\title{
Los niños del pueblo originario en el sureste mexicano y la educación intercultural bilingüe
}

Karla Del Carpio-Ovando*

*(University of Northern Colorado, Greeley, Colorado, EUA)
Resumen: En este artículo describimos la situación actual de algunos programas bilingües implementados en regiones habitadas por pueblos originarios en México. Asimismo se discuten las deficiencias y las graves consecuencias que dichos programas han tenido en los pueblos originarios al igual que en sus lenguas y culturas. En este texto se enfatiza la importancia, el valory la urgencia de otorgar una educación intercultural bilingüe de calidad a los niños indígenas mexicanos es por ello que en el presente se proporcionan las características de verdaderos programas bilingües y se compara el tipo de educación bilingüe implementada a niños indígenas mexicanos con el programa de inmersión canadiense (o mejor conocido como programa de inmersión en francés) por ser un modelo educativo que podría usarse y adaptarse al contexto de México.

Palabras claves: Educación bilingüe. Educación indígena. Niños. México. 


\section{PRESENTACIÓN}

Antes de adentrarnos al tema en cuestión, es necesario contextualizar la situación de los pueblos indígenas en México para así poder tener un mayor entendimiento de la situación actual de las comunidades indígenas al igual que de sus lenguas y culturas y el tipo de educación que han recibido centrando la atención en los niños de los pueblos originarios, por ejemplo, en los niños tsotsiles del sureste mexicano.

\section{LAS COMUNIDADES INDÍGENAS MEXICANAS, SUS LENGUAS Y CULTURAS}

López (2009) nos recuerda que en el contexto latinoamericano la nación mexicana puede destacarse por ser uno de los países con mayor número de habitantes indígenas con alrededor de 10 millones. Dicha presencia es reconocida en el artículo 2 de la Constitución Política de los Estados Unidos Mexicanos (MEXICO, 2014) documento en el que se describe al país como una nación única e indivisible además de contar con una:

Artículo $2^{\circ}$.... composición pluricultural sustentada originalmente en sus pueblos indígenas que son aquellos que descienden de poblaciones que habitaban en el territorio actual del país al iniciarse la colonización y que conservan sus propias instituciones sociales, económicas, culturales y políticas, o parte de ellas ( $p .4)$.

En el mismo artículo se reconoce y se garantiza el derecho de los pueblos y las comunidades indígenas a la libre determinación y, en consecuencia, a la autonomía para: "decidir sus formas internas de convivencia y organización social, económica, política y cultural” (MEXICO, 2014, p. 5) y para "preservary enriquecer sus lenguas, conocimientos y todos los elementos que constituyan su cultura e identidad" (MEXICO, 2014, p. 5).

Gracias a sus comunidades indígenas es posible decir que México es una nación rica lingüística y culturalmente. El INALI (2015) en su Catálogo de Lenguas Indígenas Nacionales indica que en el país existen aproximadamente “68 lenguas que tienen 364 variantes lingüísticas pertenecientes a 11 familias" (INALI, 2015, p. 15) siendo los estados de Oaxaca, Chiapas, Puebla, Yucatán, Guerrero, Quintana Roo, Hidalgo, Campeche y San Luis Potosí los que tienen mayor número de hablantes de lenguas indígenas: náhuatl, maya, mixteco, tsotsil, tseltal, zapoteco, otomí, totonaco y mazateco (INALI, 2015). El español o castellano es el idioma oficial del país; sin embargo, las lenguas originarias también son reconocidas por el gobierno mexicano lo cual se 
puede observar en la Ley General de Derechos Lingüísticos de los Pueblos Indígenas (MEXICO, 2003). En el artículo 1ํㅜ de esta ley general se destacan el reconocimiento y la protección de "los derechos lingüísticos, individuales y colectivos de los pueblos y comunidades indígenas" (MEXICO, 2003, p. 1) al igual que el del uso y desarrollo de las lenguas de dichas comunidades ya que son "parte integrante del patrimonio cultural y lingüístico nacional” (MEXICO, 2003 , p. 1) y son consideradas como una de las expresiones fundamentales de la pluriculturalidad de México. Como se mencionó anteriormente el sureste mexicano, el estado de Chiapas, por ejemplo, se caracteriza por su población indígena es por ello que en el presente manuscrito se centra la atención en dicha región.

El sureste mexicano: Chiapas

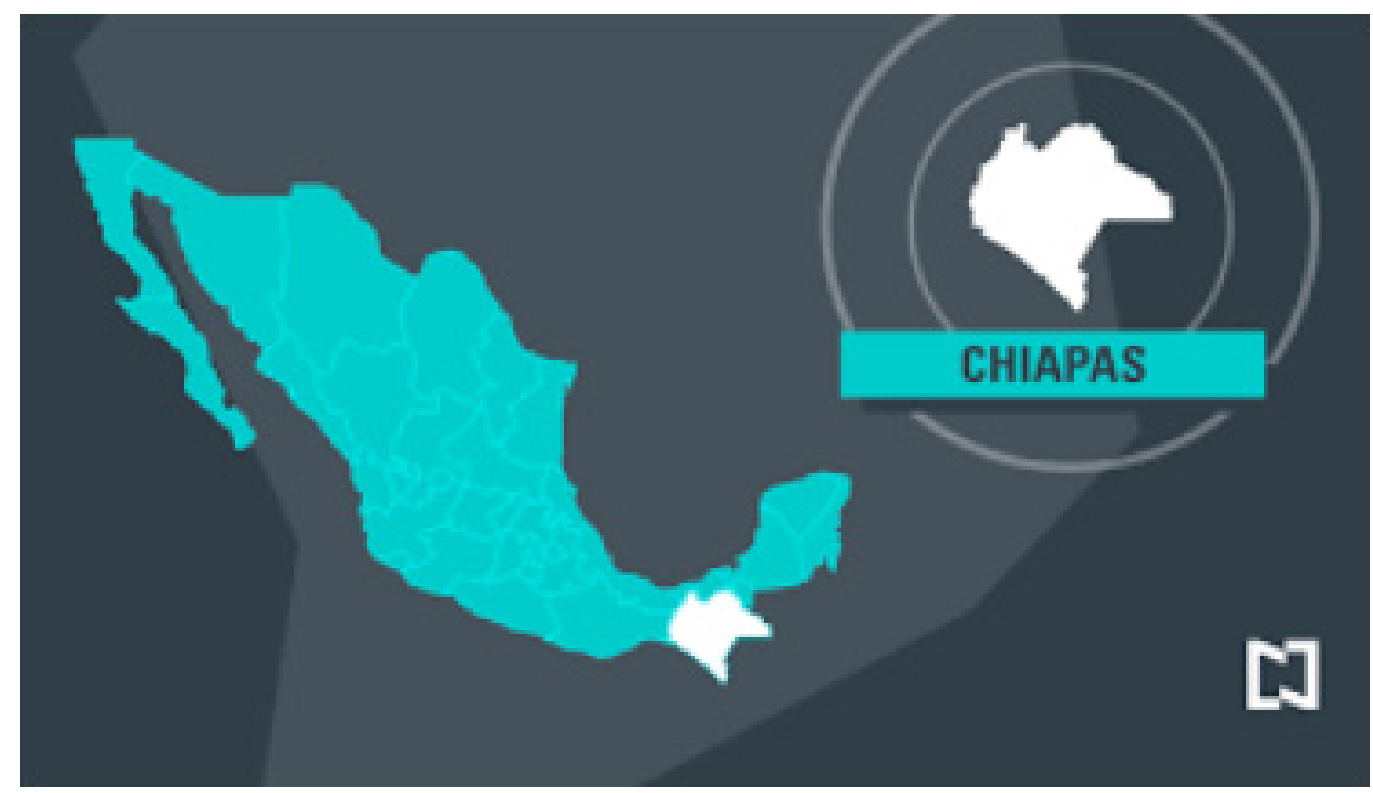

Imagen 1. Mapa del estado de Chiapas (GOOGLE, s.f.)

De acuerdo con la Secretaría de Trabajo y Previsión Social (2017), Chiapas cuenta con una población de 5,358,291 y es uno de los estados con mayor flora y fauna del país. Con respecto a sus pueblos originarios, el Gobierno del Estado de Chiapas (2014) destaca que en la región hay alrededor de 322.274 personas indígenas y 8 grupos lingüísticos tales como los tseltales, tsotsiles, choles, zoques, tojolabalels, kanjobales, mames y chujs (GUITART, 2006).

Históricamente y todavía en la actualidad las comunidades indígenas viven en una situación caracterizada por la pobreza, la discriminación y por la falta de atención por parte de la sociedad dominante. Son varios los pueblos originarios en Chiapas que se han autodenominado "los olvidados" ya que es así cómo se sienten en su propio país además de que muchas veces sus 
derechos humanos, lingüísticos y culturales han sido violados; situación que también se ha dado en la escuela. Esta realidad es una de las razones que ha motivado la realización de estudios etnográficos en escuelas primarias bilingües en español y en la lengua tsotsil para así poder comprender cómo es el tipo de educación que reciben los niños indígenas tsotsiles en los Altos de Chiapas, por ejemplo, en el municipio de Chenalhó (DEL CARPIO K., 2012).

Imagen 2. Niña indígena mexicana (GOOGLE, s.f)

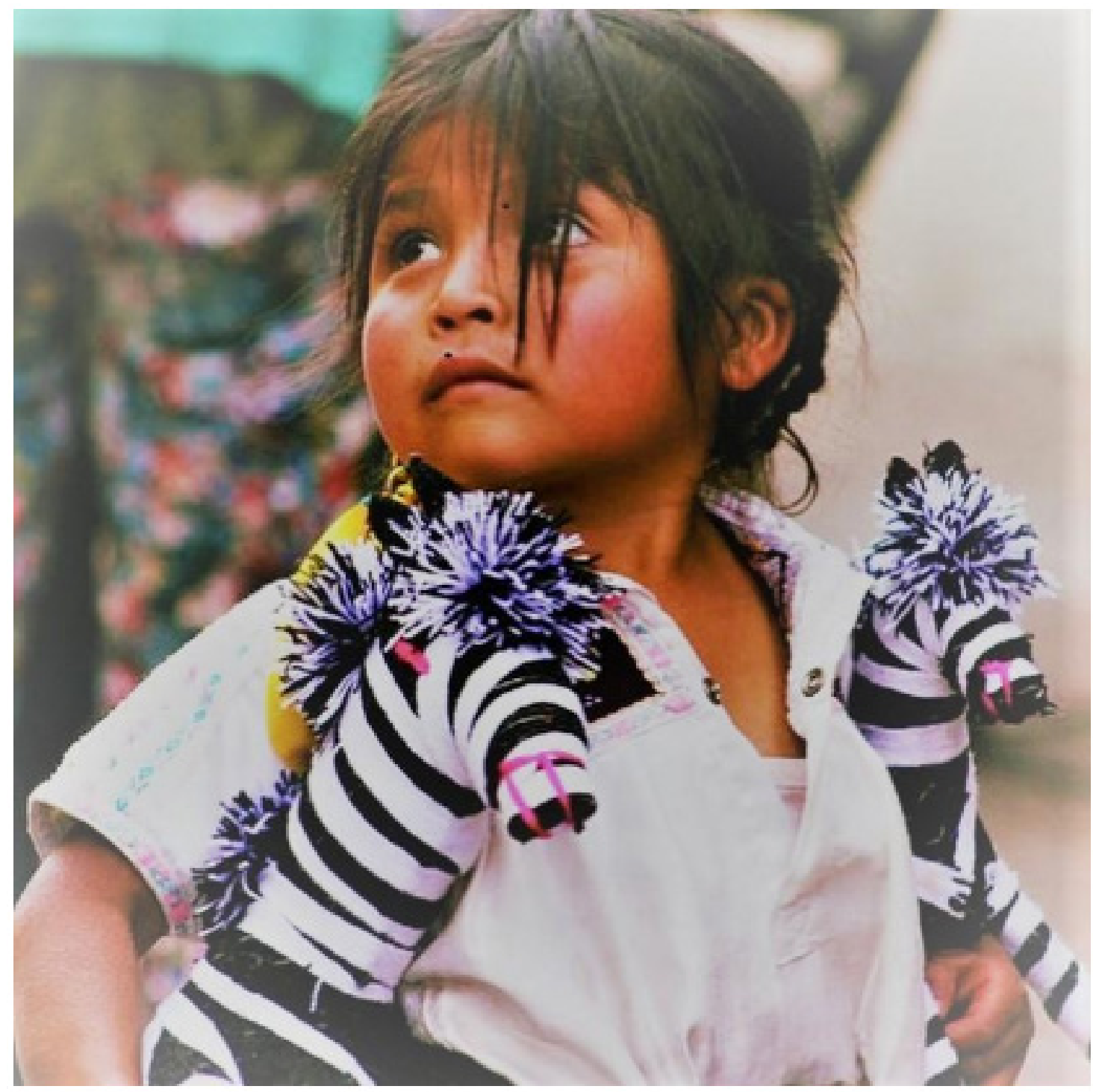

Gracias a este estudio fue posible conocer de forma cercana cómo se pone en marcha un programa "bilingüe" y cómo los estudiantes de primero y sexto grado, sus profesores y los padres de familia perciben la lengua indígena y el castellano. Se encontró que existen actitudes positivas y negativas hacia ambas lenguas pero el idioma que se considera de mayor utilidad es el español lo cual en parte se debe al énfasis que se le ha dado al idioma castellano a través de la promoción de un bilingüismo sustractivo donde solamente se 
usa la lengua materna del niño indígena en conjunto con la segunda lengua (el español en este caso) en los primeros años escolares para después darle mayor uso e importancia a ésta última (DEL CARPIO, 2012).

Básicamente un programa bilingüe ha sido basado en un enfoque monolingüe donde el castellano es la lengua dominante al igual que la lectoescritura en dicho idioma. Si bien es cierto el docente juega un papel fundamental en la implementación de un programa bilingüe; sin embargo, por lo general, los maestros encargados de dicho programa no reciben la capacitación necesaria para saber cómo enseñar con un enfoque intercultural bilingüe además de que tampoco se les brindan los materiales en español y en la lengua indígena y el contenido de los libros no refleja la realidad del niño indígena lo cual disminuye la posibilidad de un aprendizaje significativo ya que no es fácil que puedan identificarse con el tema que se les enseña. Esta situación pone en desventaja al niño del pueblo originario en comparación al niño de la sociedad dominante es por ello posible decir que los niños tsotsiles en este caso no gozan del privilegio al cual tienen acceso los infantes de áreas más desarrolladas de la nación.

En base al estudio realizado y a otras investigaciones; es posible decir que la educación bilingüe puede ser una herramienta de inclusión o un arma que disminuye o aniquila la diversidad lingüística y cultural de una nación. Esto depende del propósito del programa y de su diseño. Por ejemplo, si tiene como objetivo la asimilación del pueblo indígena a la sociedad mayoritaria puede decirse que el programa bilingüe es usado como un arma, pero si por el contrario tiene como objetivo la promoción de un bilingüismo aditivo que contribuya a la defensa de los derechos lingüísticos de los niños tsotsiles, dicho programa puede ser visto como una herramienta poderosa. Asimismo cuando éste es el objetivo, la experiencia del niño indígena en la escuela puede ser positiva ya que incrementan las posibilidades de que se sienta involucrado en su proceso de aprendizaje y que tenga un rol más activo si se le enseña en su lengua materna y además se le enriquece con una segunda lengua en este caso con el español. Desafortunadamente éste no siempre es el caso ya que se ha olvidado que las comunidades originarias enriquecen lingüística y culturalmente al estado y a la nación. Muchos de estos grupos continúan preservando sus tradiciones, valores y lenguas. No obstante, algunos aspectos culturales de los pueblos originarios han sido modificados, por ejemplo, en la actualidad muchas son las jóvenes indígenas que ya no tienen la costumbre de bordar lo cual es una práctica bastante común en las generaciones de mayor edad en la comunidad tsotsil en Chiapas. Sin 
embargo, estas jóvenes admiten que "les gusta observar cuando la madre o las hermanas mayores realizan textiles lo cual es curioso de notar y es que las niñas a pesar de que ya no aprenden el oficio consideran que cuando sean mayores y tengan hijas les gustaría que ellas sí aprendieran a hacer textiles" (DEL CARPIO, P., 2013, p. 12).

Otro aspecto cultural que ha cambiado es el uso de la vestimenta tradicional en las nuevas generaciones, por ejemplo, ahora a muchos niños indígenas se les pide que usen uniformes lo cual se puede observar en la entrevista realizada a la maestra Rosario (seudónimo) durante la investigación etnográfica realizada en la escuela bilingüe en Chenalhó, Chiapas (DEL CARPIO, 2012); estudio al que hice referencia anteriormente. Rosario nos comentó:

Rosario: Ahora las niñas usan uniformes. Les pedimos que usen faldas modernas. Estaban acostumbradas a usar sus nahuitas (falda tradicional indígena) y su ropa tradicional, pero la escuela está promoviendo que todo esto desaparezca.

Lo mismo se puede observar en la situación de la lengua indígena en la escuela. Al respecto Rosario nos dijo:

Rosario: Estamos dominados, sólo tenemos nuestra lengua es lo único que nos queda...y con el programa que nos mandan que dice que sólo debemos usar 100\% español... estamos desapareciendo.

Dicha situación ha sido causada por diversos factores, es decir, son varios los agentes que han afectado la situación de las lenguas y culturas indígenas de Chiapas siendo la escuela y la educación bilingüe dos de ellos. Por tal motivo, se considera importante describir algunas de sus características y sugerir formas de cómo mejorar dicho tipo de educación lo cual se expone en la siguiente sección.

Retos en la educación bilingüe

Muchos de los programas bilingües implementados en regiones habitadas por pueblos originarios en México y en otros países de América Latina han contribuido a reafirmar la división entre la sociedad mestiza e indígena ya que como se mencionó con anterioridad uno de los propósitos de este tipo de educación ha sido el de la asimilación; un tipo de educación que ha segregado y aislado al niño indígena dirigiéndolo a la timidez, introversión y a una falta de participación activa en las actividades del aula y de la escuela en general.

Cabe destacar que los cambios ideológicos en México en realidad no 
han tenido un impacto significativo en la creación e implementación de la educación bilingüe para niños indígenas. En teoría las lenguas y culturas originarias han logrado alcanzar un lugar oficial lo que se puede observar en el hecho de que ahora tienen un espacio en la escuela; institución en la que antes estaban prohibidas. No obstante, contar con un lugar oficial no es suficiente ya que esto no garantiza la preservación y promoción de las lenguas y culturas de los pueblos indígenas, es decir, reglamentar de manera oficial que éstas tienen un espacio en un ámbito educacional no quiere decir que en realidad se consideren en el proceso de enseñanza y aprendizaje. Además la preservación de las lenguas y de las tradiciones de las comunidades indígenas no debe reducirse solamente al espacio escolar sino que también deben ser integradas a otros ámbitos formales tales como los gubernamentales.

De acuerdo con Galván (2007) la educación bilingüe no ha respetado o promovido el respeto a la diversidad. Tampoco ha ayudado a fortalecer la riqueza lingüista de la nación (GALVÁN, 2007). Uno de los problemas es que este tipo de educación no ha sido basada en la realidad del niño indígena sino en aquélla de la sociedad dominante. Un ejemplo de ello son los contenidos de los libros de texto que reflejan la realidad del niño de la ciudad; la del niño mestizo, no la del niño indígena lo cual dificulta la tarea del maestro a la hora de usar dicho material (DEL CARPIO, 2012).

Otro problema que ha obstaculizado la implementación de programas bilingües es que a los niños indígenas se les habla y enseña en una lengua ajena a ellos. Consecuentemente en estos pequeños se han desarrollado sentimientos de timidez y reticencia (HORNBERGER, 2006). Por ejemplo, en Sudamérica, se ha encontrado que muchos pequeños pierden la voz en el aula, es decir, no hablan, no participan, son tímidos y se aíslan porque la lengua que se usa en dicho espacio es ajena a ellos (HORNBERGER, 2006). Estos niños, pues, no se involucran en las actividades escolares lo cual hace que adopten un rol pasivo en el colegio. No obstante, recuperan su voz y su forma de ser cuando regresan a casa. En otras palabras, muchos niños indígenas se vuelven ajenos a ellos mismos (a su personalidad real) cuando van a la escuela ya que en dicha institución se les quita la voz. Necesitan, pues, regresar a casa para poder así recuperarla y tener la libertad de ser quiénes son ya que es en este espacio donde se les habla en la lengua que conocen y donde pueden comunicarse en la lengua con la que se identifican; la lengua en la que se sienten cómodos por ser la que dominan y la hablada por la mayor parte de sus familiares. Esto también ejemplifica los espacios que han sido asignados a las lenguas minoritarias y a la lengua dominante (DE JONG, 2011). 
Esta situación confirma lo que diversos estudios de tipo etnográfico han encontrado sobre este tema. Paciotto (2000) nos señala que las lenguas de los pueblos originarios, por lo general, han sido consideradas con poco prestigio. Por tal motivo, han sido restringidas a la comunidad y al espacio oral comunicativo informal. Por lo contrario, los espacios sociales más amplios han sido invadidos por la lengua dominante en este caso por el castellano. En México las lenguas indígenas han sido asociadas con la población e identidades desfavorecidas lo que ha provocado que algunos hablantes de lenguas indígenas prefieran distanciarse de su propia lengua y cultura para así adoptar las dominantes (DORIAN, 1998); situación que ha sido reforzada en la escuela.

Es grave ver que a los niños indígenas se les ha enseñado a través de una práctica silenciadora que los marginaliza y oprime. Es por ello esencial que los docentes en las instituciones bilingües reciban la formación necesaria para poder así enseñar con un enfoque intercultural bilingüe el cual debe afirmar la identidad del alumno en lugar de marginarlo.

La ausencia de la lengua materna de los niños indígenas en su proceso de enseñanza aprendizaje es un problema con consecuencias destructivas e injustificables sobre todo porque que es ilógico esperar que estos pequeños participen de forma verbal en el salón; es irreal querer que se comuniquen en una lengua que no conocen. Como bien lo cuestiona Hornberger (2006) "¿Después de todo, quién puede hablar en una lengua que simplemente no conoce?” (p. 278). Por tal motivo, uno de los factores más significativos en el comportamiento pasivo de los niños que han pasado por este tipo de programa es que a estos pequeños se les habla y enseña en una lengua que no dominan o que simplemente no conocen, es decir, no se les instruye en su lengua materna sino en la lengua dominante; en la lengua del mestizo. No hay que olvidar que esta práctica viola los derechos humanos lingüísticos de estos pequeños y al imperialismo lingüístico. Se olvida, pues, que el propósito natural de la escuela y del proceso educativo es el de educar y de integrar; no el de oprimir, discriminar o segregar. La escuela debe "contribuir a la formación de niñas y niños que logren el desarrollo de sus capacidades, competencias, actitudes y valores, que les permita conocer su realidad, comprenderla, y actuar en ella para transformarla” (HERNÁNDEZ, 2009, p. 3). En resumen, se necesita una escuela para la vida; para la vida de hoy.

La realidad vivida por los niños indígenas muestra la urgencia de cambiar la dirección de la escuela y del proceso educativo. No queremos que los niños 
indígenas, ni que ningún niño o estudiante en general, pierdan su lengua; elemento esencial de cultura para lograr la propia identidad (RíOS, 2001). No queremos que estos infantes pierdan los elementos que son valiosos y esenciales para ellos tales como sus tradiciones y sus modos de ser, de percibir el mundo; su cosmovisión. De suceder esto, estaríamos perdiendo gran parte de la riqueza de la nación y tendríamos una sociedad aún más fracturada es por ello necesario implementar programas bilingües de calidad y alejarnos de intentos fallidos o de modelos educativos que solamente se han aprovechado de la lengua indígena para luego reemplazarla por la lengua mayoritaria lo que nos recuerda a los famosos programas de educación bilingüe de transición en los cuales al inicio a los estudiantes se les instruye en su lengua materna pero después de forma gradual se utiliza la segunda lengua hasta ser esta última la única lengua de instrucción (DE JONG, 2011). Es necesario alejarnos de una educación que promueva un bilingüismo sustractivo donde la lengua materna del alumno solamente se utiliza mientras aprende la lengua dominante; proceso que termina en un monolingüismo en dicha lengua (FAJARDO, 2011). No hay que olvidar que el objetivo de la educación bilingüe debe ser el de enriquecer al estudiante; no el de empobrecerlo. Por lo tanto, se debe promover una educación bilingüe de mantenimiento y desarrollo (DE JONG, 2011; FAJARDO, 2011) en el cual se conserve la lengua del alumno. Fajardo (2011) nos explica que en este tipo de programa:

\footnotetext{
La lengua materna se mantiene en el resto de la vida escolar para potenciar el bilingüismo aditivo, ya que la sustitución de la segunda lengua no sustituye a la primera. El marco político es el pluralismo cultural, orientado a la aceptación positiva de la diversidad y al desarrollo integral del alumno bilingüe. La lengua materna y la cultura de la cual es portadora, tiene un papel fundamental como un recurso pedagógico y como depositarias de saberes, conocimientos, actitudes y valores capaces de enriquecer la educación de todos los educandos (FAJARDO, 2011, p. 22).
}

Es este tipo de educación, pues, el que necesitamos implementar y difundir en las comunidades de los pueblos originarios para lograrlo uno de los pasos que hay que dar es el de tomar acción para mejorar las actitudes hacia las comunidades indígenas, sus lenguas y culturas. 


\section{ACTITUDES}

Las actitudes juegan un papel fundamental en la situación de una lengua. Autores como Eagly y Chakine $^{1}$ (1993, p. 1, apud CUETO; ANDRADE; LEÓN, 2002) recalcan que una actitud es "una tendencia a evaluar una entidad con algún grado de aceptación o rechazo, normalmente expresado en respuestas cognitivas, afectivas o conductuales". Una actitud motiva una predisposición ya sea favorable o desfavorable. Desafortunadamente hay docentes en escuelas bilingües que muestran tener actitudes negativas hacia la lengua indígena lo que los motiva a no enseñarla, preservarla o promoverla lo cual perjudica la situación de la lengua originaria. Estas actitudes desfavorecedoras hacia la lengua indígena surgen como resultado de las actitudes que existen hacia los hablantes de ésta lo que De Jong (2011) denomina the language transfer ideology para explicar la relación estrecha que hay entre los hablantes y su lengua lo que significa que si una comunidad goza de prestigio (de un alto estatus social) como consecuencia su lengua gozará del mismo prestigio. Lo mismo sucede cuando a una comunidad se le asigna un bajo estatus social como resultado a su lengua también se le es asignado dicho estatus desfavorecedor. Esto último es lo que ha sucedido con los pueblos originarios y sus lenguas lo cual ha desarrollado actitudes negativas hacia estos grupos por parte de los mestizos y de los pueblos indígenas hacia ellos mismos también subrayando así la situación de desventaja e inferioridad que se les ha otorgado a los pueblos, lenguas y culturas originarias. Por ejemplo, se ha encontrado que maestros trabajando en escuelas bilingües enfatizan el uso del español pensando que es la única lengua que les brindará oportunidades a sus alumnos y que por lo tanto la lengua indígena no necesita ser enseñada en la escuela por no tener prestigio social (DEL CARPIO, 2012). Serrano (1998) nos recalca que es común encontrar actitudes hererocentristas, que colocan "todo el énfasis en la transmisión de contenidos propios de la cultura dominante, como el etnocentrismo o regionalismo, cuando se trata de maestros mestizos, actitudes ingenuas en ambos casos. Tanto una como otra actitud constituyen negaciones del enfoque intercultural, y la función de mediador intercultural no se puede cumplir sin una curiosidad crítica" (p. 44). Es esta curiosidad la que debe desarrollarse en el docente de la escuela bilingüe.

EAGLY, A.; CHAIKEN, S. The psychology of attitudes. Fort Worth: Tex. Harcourt Brace Jovanovich College Publishers, 1993. 


\section{EL TÉRMINO EDUCACIÓN Y EL ROL DEL DOCENTE}

Autores como Platón, Herbart, Rousseau y Dewey ${ }^{2}$ nos recuerdan que el término educación tiene una connotación positiva es por ello que en la siguiente sección haremos breve referencia a dichos autores a pesar de que ninguno de ellos se enfocó en la educación bilingüe; el tema fundamental de este ensayo. No obstante, es esencial recordar que el proceso educativo (y no solamente la educación bilingüe) tienen la responsabilidad de contribuir a la vida del estudiante; objetivo que en parte se puede alcanzar a través de un docente eficiente ya que éste juega un papel primordial en el proceso educativo por ser el encargado de acompañar a los estudiantes en este proceso y de impartirles una buena educación.

Platón (s.f.) decía que:

Una buena educación es la que puede dar al cuerpo y al alma toda la belleza y toda la perfección de que son capaces. La educación es el arte de atraer y conducir a los jóvenes hacia lo que la ley dice ser conforme con la recta razón y a lo que ha sido declarado tal por los sabios y más experimentados ancianos (apud FERMOSO, 1985, p. 2).

Herbart (s.f.) describía a la educación como el arte de construir, de edificar y de dar las formas necesarias (apud FERMOSO, 1985, p. 2). Por su parte, Rousseau (s.f.) la describía como "una obra de la naturaleza de los hombres o de las cosas; el arte de educar a los niños y formar a los hombres; la educación no es sino la formación de hábitos” (apud FERMOSO, 1985, p. 2). Dewey definía a "la educación como la reconstrucción de la experiencia que se añade al significado de experiencia, y que aumenta la habilidad para dirigir el curso de la experiencia subsiguiente" (apud FERMOSO, 1985, p. 2).

Todas estas definiciones, pues, nos recuerdan que la educación debe ser un proceso enriquecedor que contribuya a la vida del alumno; que lo respete y que lo dignifique. Para ello es indispensable que el maestro cumpla con el rol que le corresponde lo que no ha sido el caso de varios docentes en escuelas bilingües. Ésta ha sido una de las dificultades que no ha permitido la implementación de una verdadera educación bilingüe además de que no existe claridad con respecto al perfil del docente ni con respecto a los logros que se quieren alcanzar con este tipo de programas; razón por la cual la calidad

\footnotetext{
Las obras de Platón, Herbart, Rousseau y Dewey no se enlistan de forma individual ya que no fue posible encontrarlas de forma separada, es decir, aparecen de forma conjunta en diversos trabajos sobre educación. Fermoso (1985) también los usa de forma grupal cuando explica la definición del término educación.
} 
de éstos es deficiente; calidad que como enfatiza Serrano (1998) nisiquiera puede ser comparada con otros programas de educación básica dirigidos a otros grupos de población. Es por ello esencial subrayar la importancia de formar al docente de educación bilingüe con el fin de que tenga conocimientos lingüísticos y culturales de ambos grupos (de su propia lengua y cultura y también de las de sus estudiantes). Serrano (1998) exalta la necesidad de que el docente tenga dominio de ambas lenguas lo cual no sucede con frecuencia:

El docente en la escuela bilingüe debe tener conocimiento tanto de su cultura como de la hegemónica así como competencia en las dos lenguas, por lo menos, en sus formas oral y escrita, y capacidad para orientar procesos de aprendizaje de y en las mismas lenguas a partir del análisis de situaciones sociolingüísticas y culturales relativamente complejas de los alumnos y de sus entornos sociales ( $p .40)$.

Por otra parte, es común que tanto la educación bilingüe como la enseñanza de segundas lenguas se visualicen como separadas o desligadas del sector educativo en general, es decir, son "tratadas" como si no fueran parte del sistema educativo nacional (DE JONG, 2011). Por lo tanto, los docentes se sienten apartados o hasta aislados del sistema como si no fueran una prioridad lo cual se puede ver en la falta de atención y capacitación que reciben. Esto también se puede observar en la escasez de materiales bilingües con los que cuentan. Hace falta, pues, profundizar con respecto a la tarea del maestro bilingüe y en los recursos que necesita. Hace falta:

Crear y fortalecer una tradición sobre formas alternativas de ser maestro propias de la Educación Intercultural Bilingüe. Para avanzar en esa línea se requiere, por supuesto, el paso de más de una generación de maestros bien formados para su campo de trabajo, pero es posible que esa generación esté apenas formándose (SERRANO, 1998, p. 42).

También es fundamental informar al docente con respecto a la importancia y el valor de la diversidad lingüística y cultural además de proporcionarle las herramientas adecuadas para saber cómo usar ambas lenguas y culturas durante el proceso de enseñanza y aprendizaje sin darle mayor prioridad o solamente enfocarse en la lengua y cultura dominantes. "El maestro indígena debe ser un ejemplo de sujeto activo y conciencia crítica con respecto a su propio pueblo" (SERRANO, 1998, p. 46). De la misma forma es preciso informar al maestro con respecto los beneficios que el bilingüismo aditivo tiene para sus alumnos. De llevarse a cabo lo sugerido en estas líneas el docente podría aumentar las posibilidades de implementar una educación bilingüe verdaderamente de calidad lo cual ayudaría a afirmar la identidad de sus alumnos. No obstante, no hay que olvidar que la creación e implementación 
de estos programas no es un sueño inalcanzable lo cual se puede ver en los programas que han sido implementados en otras naciones. Efectivamente el contexto en cada región es diferente; sin embargo, vale la pena mencionar algunos ejemplos, cómo funcionan y algunas de sus características con el objetivo de mostrar que sí es posible lograr una educación donde dos lenguas y culturas sean respetadas, preservadas y promovidas. Esto con el fin de motivar la creación de este tipo de educación en el contexto mexicano especialmente en zonas habitadas por comunidades indígenas.

\section{Programas de INMERSIÓN: EL CASO de CANAdÁ}

La educación bilingüe ha sido un medio de instrucción importante en diferentes países tales como Canadá, Japón, Estados Unidos, Paraguay y Perú. Es cierto que existen similitudes y diferencias entre los modelos bilingües que estas naciones han implementado y que un aspecto importante alrededor del sistema educativo es el contexto de cada país. No obstante, vale la pena analizar diversos programas bilingües para compararlos, contrastarlos y tomar aquellos elementos que han contribuido a la eficacia y al éxito de dichos programas. Esto con el fin de incorporar dichos elementos en modelos bilingües implementados en otras regiones reconociendo el contexto, las características y las necesidades de cada lugar y su población.

En las siguientes líneas el enfoque estará en Canadá y sus programas de educación bilingüe conocidos como programas de inmersión en francés (French Immersion Programs), los cuales han sido usados como modelos en otros países tales como Francia y España debido al éxito que han tenido en la nación canadiense (UNIVERSIDAD DE MICHIGAN, s.f.).

\footnotetext{
El propósito principal de los programas de inmersión canadienses es el de promover el bilingüismo en una comunidad global ya que considera que son muchos los beneficios que el bilingüismo puede proporcionarles a los estudiantes en su vida presente y futura. Además estos programas ayudan a expandir y a valorar la cultura francesa. Los estudiantes aprenden el mismo contenido que aprenderían en una escuela regular ya que no hay diferencia en el currículo. La única distinción es la lengua de instrucción; distinción que ha mostrado tener muchos efectos positivos en los alumnos (UNIVERSIDAD DE MICHIGAN, s.f., p. 5).
}

Los programas de inmersión son populares en la nación canadiense lo cual se puede observar en el hecho de que se encuentren disponibles en todas sus provincias. Hay aproximadamente cerca de 300.000 estudiantes en más de 2.100 escuelas en todo el país (UNIVERSIDAD DE MICHIGAN, s.f.). Existen 
3 tipos de inmersión en este modelo educativo nominados en inglés como Early (al inicio o temprano), Middle (a la mitad o en medio) y Late (tardío). Los 3 han demostrado ser eficaces no obstante el que mayor éxito ha tenido es el de Early Immersion ya que ha tenido mejores resultados que el de Late Immersion. En las siguientes líneas se describe brevemente cada tipo de programa de inmersión.

Programa de Inmersión Temprana (Early Immersion)

Este tipo de programa comienza en el kínder o en primer grado y se caracteriza por la instrucción brindada $100 \%$ en francés desde el inicio hasta el segundo grado. En tercer grado se introduce lo que se conoce como English Language Arts y la instrucción en francés se reduce al $80 \%$ y continúa disminuyendo durante el resto de los siguientes grados. Al llegar al grado 12, la instrucción se da en francés por $50 \%$ del día escolar.

Programa de Inmersión en Medio (Middle Immersion)

Éste comienza en grado 3 o 4 y usualmente empieza con una instrucción 80\% en francés. Este programa es una combinación de Early Immersion y Late Immersion es por ello que su eficacia esta entre ambos tipos de programa.

Late Immersion

Comienza en los grados 607 con una instrucción entre el $60 \%$ y $75 \%$ en francés. Las materias tales como ciencias sociales y matemáticas son impartidas en francés mientras que la clase de inglés y materias opcionales o electivas son dados en inglés. Comúnmente los estudiantes en estos programas alcanzan el mismo nivel de competencia en lectura y escritura que los alumnos en los programas de inmersión temprana; sin embargo, su expresión oral usualmente es inferior. El hecho de que los programas de inmersión en francés reciban un fuerte apoyo en Canadá es especialmente porque promueven el bilingüismo el cual ha demostrado brindar efectos positivos en las habilidades cognitivas de los alumnos lo que contradice los mitos que comúnmente se han tenido con respecto al bilingüismo, por ejemplo, cuando se dice que afecta el desempeño del hablante en una de las dos lenguas. Por lo contrario, entre más lenguas se aprendan mayores son los beneficios que se obtienen en diferentes ámbitos de la vida. 
De acuerdo con De Jong (2011), para que un programa bilingüe sea de calidad necesita contar con los siguientes rasgos; es necesario que tenga suficientes materiales en ambas lenguas, por ejemplo, con suficientes libros de texto. Asimismo la escuela también debe contar con un staff con dominio de la lengua o lenguas de instrucción y debe estar informado con respecto al bilingüismo, segundas lenguas y la implicación de éstas en el proceso de enseñanza.

Por otra parte, el programa bilingüe debe tener una articulación clara de las expectativas curriculares y lingüísticas, es decir, debe tener expectativas explicitas sobre el usos de ambas lenguas además de que debe de brindar oportunidades para el desarrollo cognitivo y lingüístico de los alumnos (DE JONG, 2011).

Otro aspecto importante para que un programa bilingüe sea de calidad es la colaboración por parte de los docentes, por ejemplo, en el uso de ambas lenguas en diversas actividades además de que deben de hacer uso de prácticas que propicien un ambiente óptimo de aprendizaje para estudiantes bilingües. De igual modo los maestros tienen que mantener altas expectativas, es decir, no deben de disminuir la competencia de ninguna de las lenguas ni los estándares académicos (DE JONG, 2011).

Los docentes también deben de usar enfoques de enseñanza actualizados que tengan como base tanto la lengua materna del alumno como la segunda lengua. Por otro lado, los profesores necesitan implementar un currículo que esté construido y que refleje las experiencias culturales de los estudiantes. De la misma forma deben implementar prácticas de instrucción que respondan a ambas lenguas y culturas (DE JONG, 2011). Aparte hay que recordar que un programa bilingüe de calidad debe estar basado en principios fundamentales que guíen las decisiones educativas a nivel local y que informen las políticas con respecto al uso de las lenguas en el aula y en la escuela en general (DE JONG, 2011). Los cuatro principios esenciales sugeridos por De Jong (2011) son el principio para lograr la igualdad educativa, el principio para afirmar las identidades de los estudiantes, el principio para alcanzar un bilingüismo o multilingüismo aditivo y el principio de integración (DE JONG, 2011). Estos principios tienen como propósito involucrar a los maestros en prácticas educativas que sean flexibles y apropiadas al contexto de sus alumnos. Un programa que promueve el bilingüismo y la lectoescritura en dos idiomas lo que significa que promueve un bilingüismo aditivo, hace uso de prácticas 
educativas que responden a la diversidad cultural de los estudiantes, es decir, utiliza prácticas que afirman su identidad y que aseguran la participación de padres del grupo minoritario y mayoritario. Igualmente estos principios buscan que el sistema de evaluación considere el desempeño de los alumnos no solamente en la lengua mayoritaria sino que también la lengua minoritaria sea tomada en cuenta en este proceso de evaluación.

\section{RETOMANDO LO ANTERIOR}

América Latina es una región con una gran riqueza lingüística y cultural gracias a sus comunidades indígenas. Un ejemplo claro de estos son los pueblos originarios en la nación mexicana; país conocido por ser uno de los que cuenta con mayor población indígena y por lo tanto con un número de lenguas indígenas bastante significativo. Sin embargo, varias de estas lenguas han estado en peligro debido a diversos factores siendo la educación bilingüe uno de ellos ya que ha privilegiado a la lengua y a la cultura mayoritarias; a los mestizos y al español. La falta de uso de la lengua indígena, por ejemplo, en los programas bilingües dirigidos a niños indígenas tsotsiles en Chiapas ha tenido repercusiones no solamente en la situación de la lengua indígena sino también en sus hablantes lo cual es grave. Se ha encontrado, pues, que cuando al niño indígena se le habla y enseña en una lengua mayoritaria; lengua que muchas veces no domina o simplemente no conoce, se fomenta en él un comportamiento de timidez, introversión y pasividad por no comprender lo que se le dice; éste es, pues, el rol que toma cuando está en la escuela. Por lo contrario, cuando regresa a casa, recupera su personalidad real y un comportamiento de comodidad y libertad porque es el contexto en el que puede ser él mismo y expresarse en la lengua que domina; en la lengua con la que se identifica; en la lengua de sus familiares. Esto nos recuerda el propósito de la educación y de la escuela que es el de preparar al alumno para la vida para lograrlo se necesita una escuela que contribuya a la formación de niños que "logren el desarrollo de sus capacidades, competencias, actitudes y valores, que les permita conocer su realidad, comprenderla y actuar en ella para transformarla. En pocas palabras: una escuela para la vida" (HERNÁNDEZ, 2009 , p. 3) para ello se necesita un maestro "abridor de mundo." Dicho de otro modo, el maestro debe trabajar.

Desde sus propias raíces y para la comprensión de la situación de sus alumnos; debe ser capaz de dialogar con los padres de familia y con otros miembros de los grupos locales y convencerlos de las bondades de la modalidad educativa en la que trabaja. Para lograrlo requiere, además de actitud, conocimiento de 
los dos campos culturales y un saber pedagógico. En cuanto a la de su grupo étnico, conviene insistir en que no basta con vivir en la cultura y hablar la lengua, sino que hay que darse cuenta de ello. De lo contrario, no se podrá ser agente crítico. En este sentido, la formación que recibe afronta el reto de convertirlo en un observador permanente y atento de su propia cotidianidad y la de los suyos, en contraposición a la creencia de que uno no necesita estudiar su cotidianidad porque ya la conoce (SERRANO, 1998, p. 51).

Lo anterior es uno de los puntos importantes para que un programa bilingüe sea de calidad además de que debe estar basado en los principios sugeridos en este artículo. Es verdad que llevarlos a la práctica requiere un proceso continuo de reflexión y acción; es un proceso lleno de retos; sin embargo, cualquier intento para promover una educación bilingüe que respete y dignifique a los estudiantes, sus lenguas y culturas vale la pena.

Existen ejemplos reales como es el caso de los programas de inmersión en Canadá que han logrado fomentar un bilingüismo aditivo enriquecedor es por ello importante analizarlo para poder comprender cómo funciona y tomar aquello que podría ser utilizado en el contexto mexicano. Estamos conscientes de que la realidad canadiense es diferente a la de los niños indígenas en México y que las condiciones de las lenguas en ambas naciones también son diversas además de que cada modelo educativo funciona de acuerdo con la realidad en el cual es implementado es por ello que nuestra intención al mostrar el ejemplo de Canadá es el de presentar otras iniciativas que han sido exitosas con el fin de considerarlas como referencia para los programas bilingües que actualmente son implementados en comunidades indígenas, por ejemplo, en zonas habitadas por la población tsotsil en el sureste mexicano.

Es cierto, pues, que son varios los retos a superar con respecto a la educación bilingüe en México pero de poder lograrlo estaríamos contribuyendo no solamente al sistema educativo sino también al fortalecimiento de la diversidad lingüística y cultural del país la cual es central en cualquier sociedad y en cualquier nación. Asimismo estaríamos haciendo valer los derechos lingüísticos y culturales de los pueblos indígenas mexicanos. No hay que olvidar que cada comunidad tiene su dignidad y valor los cuales deben ser respetados y preservados $(\mathrm{BURCH}, 2003)$. Son, pues, varios los beneficios de mejorar la educación bilingüe en vigencia por lo tanto vale la pena seguir insistiendo en el tema con el fin de sobre todo contribuir a que nuestros niños indígenas sean respetados y que reciban un tipo de educación de calidad que los respete y que promueva el desarrollo de sus habilidades lingüísticas 
y culturales. Queremos, pues, una educación donde los educadores sean artistas y la educación una obra de arte (FULLAT, s.f.); una educación que contribuya a una sociedad más justa e incluyente 


\section{Bilingual Education in the Mexican Context: Indigenous Children's Reality}

Abstract: This article describes the current situation of some bilingual programs implemented in regions inhabited by indigenous peoples in Mexico. Also, the deficiencies and the negative effects that these programs have had on indigenous communities, their languages and cultures are mentioned. On the other hand, the importance, the value and the urgency to implement quality intercultural bilingual education for Mexican indigenous children are also emphasized. This work underlines some of the key characteristics that bilingual programs need to have. In addition, the type of bilingual education Mexican indigenous children have received is compared to Canadian Immersion Programs (better known as French Immersion Programs) since some aspects of this model could be used and adapted to bilingual education in Mexico.

Keywords: Bilingual education. Indigenous education. Mexico. 


\section{Crianças de povos indígenas no sudeste do México e educação intercultural bilíngue}

Resumo: Neste artigo, descreve-se a situação atual de alguns programas bilíngues implementados em regiões habitadas por povos indígenas no México. Da mesma forma, discutem-se as deficiências e as graves consequências que tais programas tiveram nos povos indígenas, bem como em suas línguas e culturas. O texto enfatiza a importância, o valor e a urgência de oferecer uma educação intercultural bilíngue de qualidade para as crianças indígenas mexicanas. Para isso, fornecem-se as características de verdadeiros programas bilíngues e compara-se o tipo de educação bilíngue implementado para crianças mexicanas com o programa de imersão canadense (mais conhecido como programa de imersão em francês) por ser um modelo educacional que poderia ser usado e adaptado ao contexto do México.

Palavras-chave: Educação bilíngue. Educação indígena. Crianças. México. 
BURCH, S. La diversidad cultural y lingüística. 2003. Disponible en: en el sitio http://www.rebelion.org/hemeroteca/cultura/030919dc.htm. Acceso en: 25 de octubre de 2015.

CUETO, S.; ANDRADE, F.; LEON, J. Las actitudes de los estudiantes peruanos hacia la lectura, escritura, matemática y lenguas indígenas. 2002. Disponible en: http:/www2.minedu.gob.pe/umc/admin/images/menanexos/ menanexos_89.pdf. Acceso en: 23 de enero de 2016.

DE JONG, E. Foundations for multlingualism in education: from principles to practice. Philadelphia, USA: Caslon Publishing, 2011.

DEL CARPIO, K. Spanish-Indigenous bilingual education in Chenalhó, Chiapas, Mexico. 2012. Universidad de Alberta, Canadá.

DEL CARPIO, P. Precariedad y retos del sector artesanal en Chiapas, México. Segundo congreso latinoamericano de ciencias sociales: La crisis en América Latina, diferentes perspectivas y posibles soluciones, 13 y 14 de junio, 2013. Universidad de Zacatecas: México, 2013.

DORIAN, N. Western language ideologies and small-language prospects. GRENOBLE, L.; WHALEY, L. (Eds.), Endangered languages: Language loss and community response (pp.5-21). 1998. United Kingdom, UK: Cambridge University Press.

FAJARDO, D. Educación intercultural bilingüe en Latinoamérica: un breve estado de la cuestión. Liminar Estudios Sociales y Humanísticos, San Cristóbal de las Casas, México, v. 9, n. 2, p. 15-29, 2011.

FERMOSO, P. Proceso educativo e instrucción. 1985. Disponible en: http:// cholonautas.edu.pe/modulo/upload/Fermoso\%20cap\%208.pdf. Acceso en: 24 de octubre de 2016.

FULLAT, O. (s.f.). Educación. Disponible en: http://www.pensamientos.org/ pensamientoseducacion.htm. Acceso en: 22 de octubre de 2016.

GALVÁN, A. Iniciativas de ciudadanos senadores. 2007. Disponible en: 〈http:// www.senado.gob. $m x /$ index.php? ver $=s p \& m n=2 \& s m=2 \& i d=6093 \& l g=60$. Acceso en: 27 de junio de 2011.

GOBIERNO DEL ESTADO DE CHIAPAS. Instrumentos normativos para la 
formulación del anteproyecto de presupuestos de egresos. Chiapas, México: 2014. Disponible en: http://www.haciendachiapas.gob.mx/marco-juridico/ Estatal/informacion/Lineamientos/Normativos/2014/XXIII-EstadisticaPoblacion.pdf. Acceso en: 6 de marzo de 2016.

GOOGLE. Imágenes. Acceso en: 16 de agosto de 2017.

GUITART, M. Identidades culturales. Caminando hacia la igualdad y pluralidad en la Universidad Intercultural de Chiapas. En Vilá (Ed.). Lengua, interculturalidad e identidad (p.71-91). University of Girona, 2006.

HERNÁNDEZ, J. Una escuela para la vida: algunos aspectos de una intervención pedagógica de la UPN en una comunidad indígena. Revista Universitaria de la Universidad Pedagógica Nacional, v. 8, n. 10, p. 1-10, 2009.

HORNBERGER, N. Voice and biliteracy in indigenous language revitalization: Contentious educational practices in Quechua, Guarani, and Maori Contexts. Journal of language identity and education, v. 5, n. 4, p. 277-292, 2006.

INALI. Las 364 variantes de las lenguas indígenas nacionales, con algún riesgo de desaparecer. 2015. Disponible en: http://www.inali.gob.mx/ comunicados/451-las-364-variantes-de-las-lenguas-indigenas-nacionalescon-algun-riesgo-de-desaparecer-inali.html. Acceso en: 16 de agosto de 2017.

LÓPEZ, L. Reaching the unreached: indigenous intercultural bilingual education in Latin America. Paper commissioned for the EFA Global Monitoring Report 2010, Reaching the marginalized. 2009. Disponible en: 〈http://unesdoc. unesco.org/images/0018/001866/186620e.pdf . Acceso en: 4 de marzo de 2016.

MEXICO. Constitución Política de los Estados Unidos Mexicanos. Grupo editorial RAF: México, D.F., 2014.

MEXICO. Ley General de Derechos Lingüísticos de los Pueblos Indígenas. 2003. Disponible en: file://C:/Users/Karla/Downloads/LeyGeneraldeDerec hosLinguisticosdelosPueblosIndigenas2003.pdf. Acceso en: 26 de junio de 2017.

PACIOTTO, C. Learn in beauty: Indigenous education for a new century. Encontrado en la base de datos de ERIC (ED 445 868), 2000.

RÍOS, J. Identidad y cultura: la desacralización del símbolo. Simposio Internacional de Filosofía. Identidad y cultura: reflexiones desde la Filosofía, 
p. 121-142, Universidad de A Coruña, España. 2001.

SECRETARIA DE TRABAJO Y PREVISION SOCIAL. Subsecretaría de empleo y productividad laboral. (2017). Disponible en: http://www.stps.gob.mx/ gobmx/estadisticas/pdf/perfiles/perfil\%20chiapas.pdf. Acceso en: 15 de agosto de 2017.

SERRANO, J. El papel del maestro en la Educación Intercultural Bilingüe, Revista Iberoamericana de Educación: Educación, Lenguas, Culturas, v. 17, 1998. Disponible en: http://www.rieoei.org/oeivirt/rie17a04.htm. Acceso en: 23 de octubre de 2015.

UNIVERSIDAD DE MICHIGAN (s.f.). French Immersion in Canada. Disponível em: http://sitemaker.umich.edu/356.hess/an_intro_to_bilingual_education. Acceso en: 20 de octubre de 2015.

\section{SOBRE LA AUTORA}

Karla Del Carpio-Ovando es profesora asistente en el Departamento de Estudios Hispánicos de la Universidad del Norte de Colorado, ubicada en Greeley, Colorado (EE. UU.)

Correo electrónico: karla.delcarpioovand@unco.edu

Recebido: Marzo de 2017.

Aprobado: Mayo de 2017. 